\title{
CALET on-orbit operations and data analysis system at the Waseda CALET Operations Center (WCOC)
}

\author{
Shunsuke Ozawa* \\ Research Institute for Science and Engineering, Waseda University, 3-4-1 Okubo, Shinjuku, \\ Tokyo 169-8555, Japan \\ E-mail: Shunsukedaoni.waseda.jp

\section{Yoichi Asaoka} \\ Research Institute for Science and Engineering, Waseda University, 3-4-1 Okubo, Shinjuku, \\ Tokyo 169-8555, Japan, and \\ JEM Mission Operations and Integration Center, Human Spaceflight Technology Directorate, \\ Japan Aerospace Exploration Agency, 2-1-1 Sengen, Tsukuba, Ibaraki 305-8505, Japan \\ E-mail: yoichi.asaokaeaoni.waseda.jp
}

\section{for the CALET collaboration}

CALET is the long-term high energy cosmic ray observation mission on JEM-EF of the International Space Station. In order to extend measurements of electrons and gamma rays to the 10-20 $\mathrm{TeV}$ region and protons and nuclei to several hundred $\mathrm{TeV}$, the CALET calorimeter (CAL) has a thickness of 30 radiation lengths. CAL is composed of a lead tungstate (PWO) Total AbSorption Calorimeter (TASC), a tungsten-scintillating fiber IMaging Calorimeter (IMC), and a plastic scintillator CHarge Detector (CHD).

The data taken by CALET are passed to the ISS and sent to the ground immediately via NASA and JAXA data relay links. At JAXA's Tsukuba Space Center, a ground operation system (JAXAGSE) then transmits the data to the Waseda CALET Operations Center (WCOC) at Waseda University. Monitoring of the instrument's observation status has been performed since October 2015 at JAXA. Simultaneously, the CALET science team monitors the scientific mission and the data transmission on a 24-hour 7-day basis in WCOC.

Monitoring at the WCOC is performed by Quick Look software developed at Waseda. When the Quick Look GUI detects observed values exceeding predetermined thresholds, an alarm is issued. In order to provide flexibility for the scientific operation, the CALET observing schedule is updated daily. In addition, the observation data file is processed for transmission to the science team. Raw (Level-0) data processed at JAXA-GSE are converted to engineering (Level-1) data for distribution to the international CALET collaboration. Finally, individual detector temperature dependences, time variations, etc. are included in the final (Level-2) data which are used for detailed science analysis.

35th International Cosmic Ray Conference - ICRC2017

10-20 July, 2017

Bexco, Busan, Korea

${ }^{*}$ Speaker. 


\section{Introduction}

The CALorimetric Electron Telescope (CALET) project primarily aims at discovery of nearby cosmic-ray accelerators and search for dark matter by precisely measuring electron and gammaray spectra over a wide energy range from $\mathrm{GeV}$ to TeV[?]. CALET is a high performance particle detector equipped with a thick large-area calorimeter installed in August 2015 on the Exposed Facility of the Japanese Experiment Module (JEM-EF) on the International Space Station (ISS). It will perform a long term observation for two years extendable to 5 years or more and has been collecting data since October 2015[?].

The CALET calorimeter (CAL) is composed of a lead tungstate (PWO) Total AbSorption Calorimeter (TASC), a tungsten-scintillating fiber IMaging Calorimeter (IMC), and a plastic scintillator CHarge Detector (CHD). The calorimeter of 30 radiation-length thickness completely absorbs electron shower energy in the $\mathrm{TeV}$ energy range and separates electrons from the overwhelming number of protons using the difference in shower development in the fully-active and thick calorimeter. The CALET structure and CAL schematic are shown in Figure ??. Longterm ob-
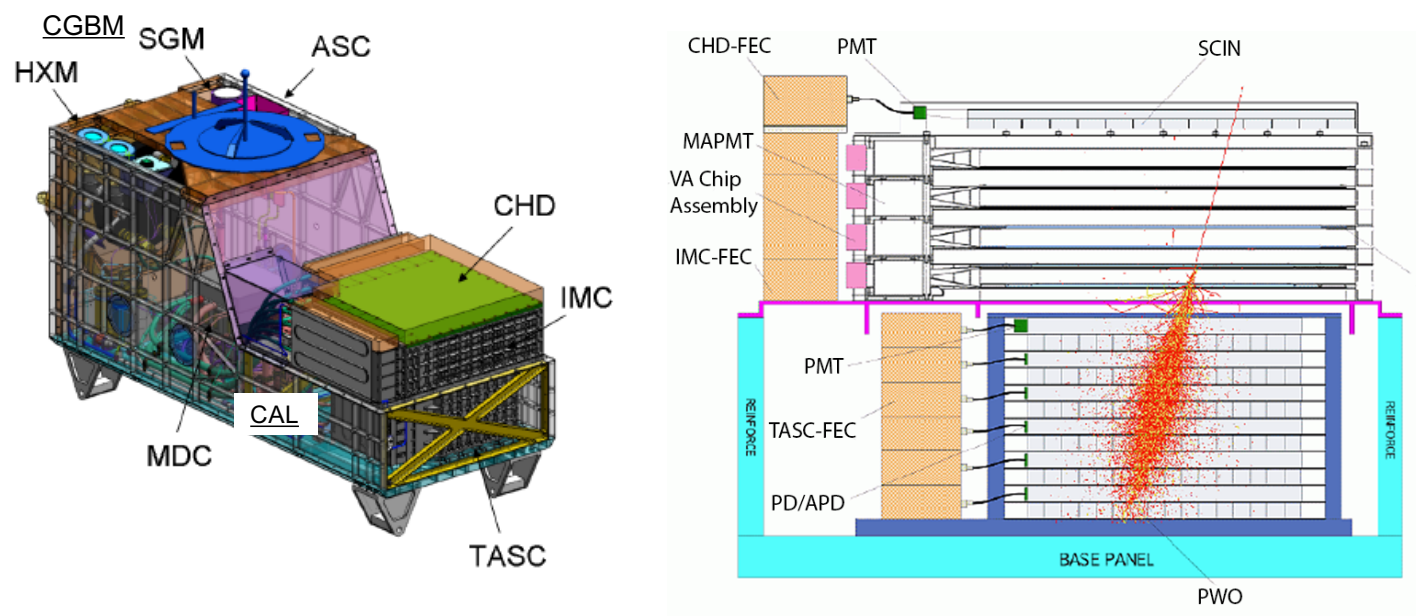

Figure 1: Structural drawing of CALET installed on the JEM-EF. The CAL structure scheme is shown on the right. Shower image shows a $1 \mathrm{TeV}$ electron created by Monte-Carlo simulation.

servation with a large-area detector is enabled by observation onboard ISS. By combining these features, it becomes possible to precisely measure the total electron spectrum up to $20 \mathrm{TeV}$ for the first time. The main components of cosmic rays such as protons, helium and heavier nuclei can be measured up to PeV. Including electrons and gamma-rays, CALET gives unique observations by extending the previous limits of direct measurements.

The trigger decision in CALET is based on the coincidence of trigger counter signals which are generated by discriminators on the analog signals from the detector components, i.e., each of CHD $\mathrm{X}$ and Y, IMC X1-X4, Y1-Y4, and TASC X1 generates lower discriminator signals. The signals from two IMC fiber layers are processed by a single front end circuit, and so each axis has only four trigger counter signals. Three trigger modes are possible in CALET. The High Energy shower trigger (HE) selects shower events with energies greater than $10 \mathrm{GeV}$, while the Low Energy shower trigger (LE) selects shower events with energies greater than $1 \mathrm{GeV}$. The Single Trigger (Single) is 
dedicated to acquiring data from non-interacting particles for the purposes of detector calibration. The pedestal trigger is used to measure the electronic offset for each readout channel.

The scientific operation of CALET is performed using a scheduled command file in which a time stamped command sequence is described. While the most important task in steady operation is to continuously take high energy shower trigger data to precisely determine the electron spectrum well into the $\mathrm{TeV}$ region, it is also necessary to take calibration data such as pedestal and penetrating particle data to calibrate MIP signals using the single trigger mode. In addition, $\mathrm{GeV}$ gamma-ray observations synchronized to the GRB trigger issued in the CGBM detector, ultra heavy nuclei data taking[?], low energy electron data taking at high latitude, low energy gamma-ray data at low latitude, and other physics targets are also interesting as long as they do not affect the primary physics target of CALET. It is important to maximize the science outcome of CALET by means of efficient observations well planned in advance.

\section{Flight data transfer}

The observation data obtained with CALET onboard ISS is transferred to JAXA. To operate and to monitor CALET, the JAXA Ground Support Equipment (JAXA-GSE) at JAXA Tsukuba Space Center (TKSC) and Waseda CALET Operations Center (WCOC) in Waseda Univ. have been established. The real-time data received by JAXA-GSE are immediately transferred to WCOC.

Scientific raw data are also transferred to WCOC on an hourly basis after time-order correcting and complementing replay data at JAXA-GSE. For the CALET project which aims at unique scientific goals by challenging the boundary of statistics and accuracy, it is very important to maintain detector performance and to carry out observation with high efficiency during the long term operations. WCOC's responsibility includes the following:

- Real-time monitoring and operations

- Operations planning

- Processing raw scientific data (Level-0) to Level-1 data

The data flow of CALET is shown in Figure ??. There is an interface to JAXA-GSE corresponding to each role of WCOC, making it possible to communicate with CALET onboard ISS. A data transmission speed of $600 \mathrm{kbps}$ usually is possible from ISS and between ground equipment connected by the Internet. The communication status of the data transmission is monitored in WCOC, the soundness of real-time data and Level-0 file are always checked.

Level-0 files delivered to WCOC are converted to Level-1 data gradually, and are used for a diagnostic of equipment and observation status.

\section{Real-time monitoring at WCOC}

To monitor the observation status of CALET in real-time, a quick look (QL) system which consolidates and visualizes cosmic-ray event data and housekeeping data was developed. Since a large amount of data must be monitored in real-time in a comprehensive manner, it is necessary to summarize the data and to detect malfunctions automatically. By utilizing simulated CALET 


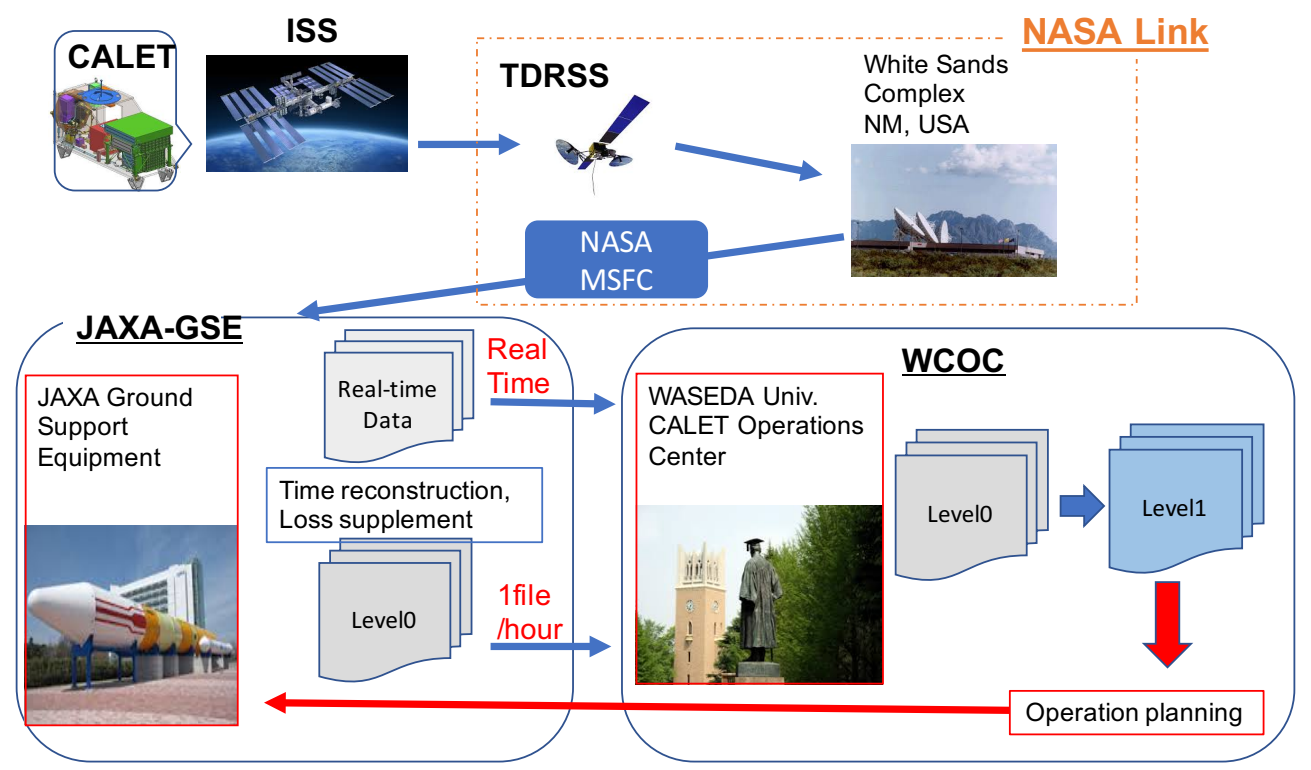

Figure 2: The data flow of CALET. Raw data are transmitted to WCOC as soon as possible for real time monitoring of the observation. And Level-0 data are translated to Level-1 data for quick analysis.

telemetry data, the QL systems were developed on a simulated situation of on-orbit operation. An operator is always present at WCOC, and the science observation status of the system is summarized for 24 hours by watching the real time data forwarded from JAXA-GSE. Quick Look (QL) uses a monitor in each PC in WCOC to visualize it immediately.

Figure ?? shows a part of the QL displays. This window displays the ISS Orbit and command schedule. The expected orbit of ISS is drawn on the map, and the timing of command execution is indicated. The color classification on the map is an indication of the trigger rate, and is devised in order to compare with the command execution time easily. Additionally event display, temperature trend of each part, the trigger rate of detectors, HV status and more are included in the QL system.

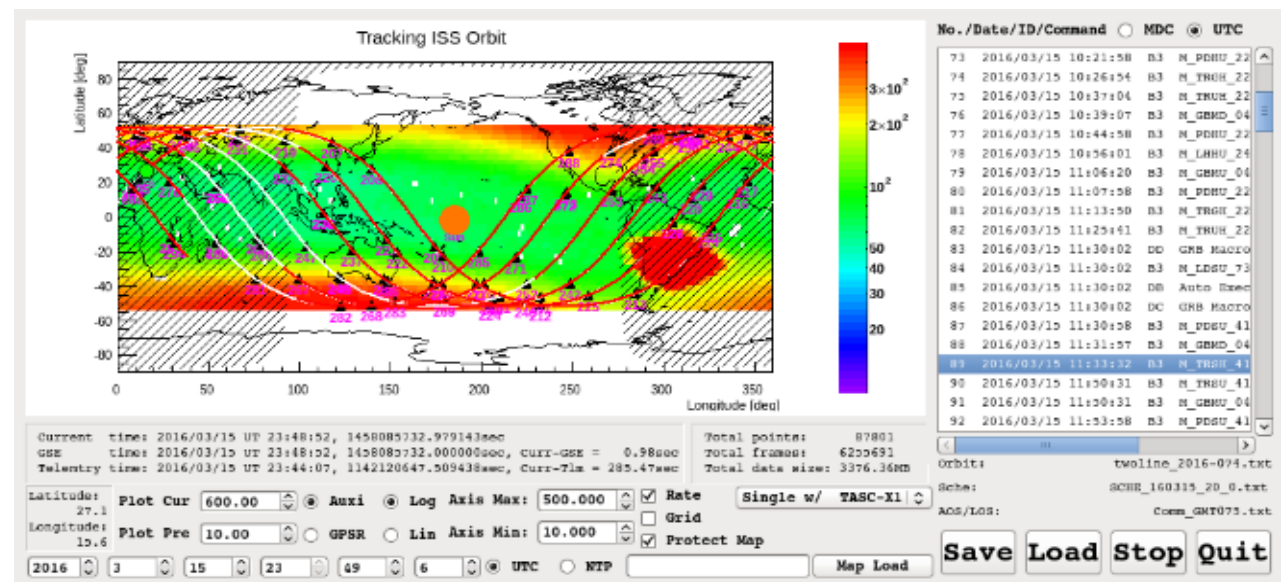

Figure 3: User interface of QL software for monitoring ISS orbit. The timing for command execution are indicated on ISS track. The orbit information, information on AOS/LOS and the schedule file are read and updated every day. The color map indicates the trigger rate of TASC X1 on this figure. 
The display being utilized to monitor the equipment and communication comprehensively is called "Summary Display". It is included to check the observation status, and when detecting abnormality, it is indicated to an operator by color and sound. Currently in the stable operation phase, the CALET science team monitors the scientific mission and the data transmission on a 24-hour 7-day basis in WCOC, utilizing the QL system.

\section{On orbit operation planning}

Planning the trigger conditions for various parts of the orbit takes account of geomagnetic latitude with the goal of efficient observation of low-energy particles and calibration data while achieving the highest possible statistics for $\mathrm{TeV}$ electrons. It requires taking account of the dead time of $\sim 5$ ms per event.

The following modes are combined in a schedule command file.

- High Energy Shower observation:

All electrons and high energy shower phenomena of gamma rays and nuclei are acquired. The high energy shower observation is always activated since this is the trigger mode for the main objectives of CALET.

- Low Energy electron observation:

For electron data of $1 \mathrm{GeV}$ region, this mode can acquire the low energy data efficiently when the geomagnetic cutoff is low. It is activated for $90 \mathrm{~s}$ at the highest geomagnetic latitude in the north and south region.

- Low energy Gamma-ray observation:

Using the geomagnetic cutoff for charged particles, low energy gamma ray data is acquired efficiently. It is activated in the low geomagnetic latitude region when the geomagnetic latitude is below $20^{\circ}$ except for the South Atlantic Anomaly (SAA).

- Single run:

To check the equipment gain and stability, we collect proton/helium events selectively. It is normally activated during two consecutive orbits ( 3 hours) per day to collect enough statistics to monitor the gain stability.

- Ultra Heavy nuclei observation:

A dedicated trigger mode to acquire ultra heavy nuclei penetrating CHD and the upper four layers of IMC is implemented. While the main target of the trigger mode is nuclei of $Z>26$, the trigger threshold is loose enough to trigger $\mathrm{Z} \geq 12$ nuclei. The ultra heavy observation is also always activated because of low trigger rate.

- Pedestal run:

Pedestal data are periodically acquired at the rate of 100 events per every 23 minutes.

This file also includes CGBM control commands to protect the detector from high radiation at high geomagnetic latitude and SAA. Observation operation is carried out by the schedule command file. This file is sequenced with the execution time taking into account the ISS position and Acquisition 
and Loss of Signal (AOS/LOS). The schedule command is created making use of recently observed data and is renewed every day semi-automatically in WCOC.

\section{Data analysis system}

The analysis scheme of CALET is shown in Figure ??. The scientific raw data called CALET Level-0 data are generated at JAXA and transferred to WCOC. In the Level-0 data, although the time order is corrected and missing data are complemented, it is a simple format where received packets are only connected. In WCOC, we convert them into Level-1 data which is the base data for scientific data analysis and distribute them to the international CALET collaboration for scientific analysis. Quick analyses based on both Level-0 and Level-1 data are performed and their results are used as feedback for better operations planning and real-time monitoring. Level-1 data include all event, housekeeping, rate, and ancillary data records with timestamps corrected to UTC. The housekeeping temperatures, voltages and currents are converted to physical units.

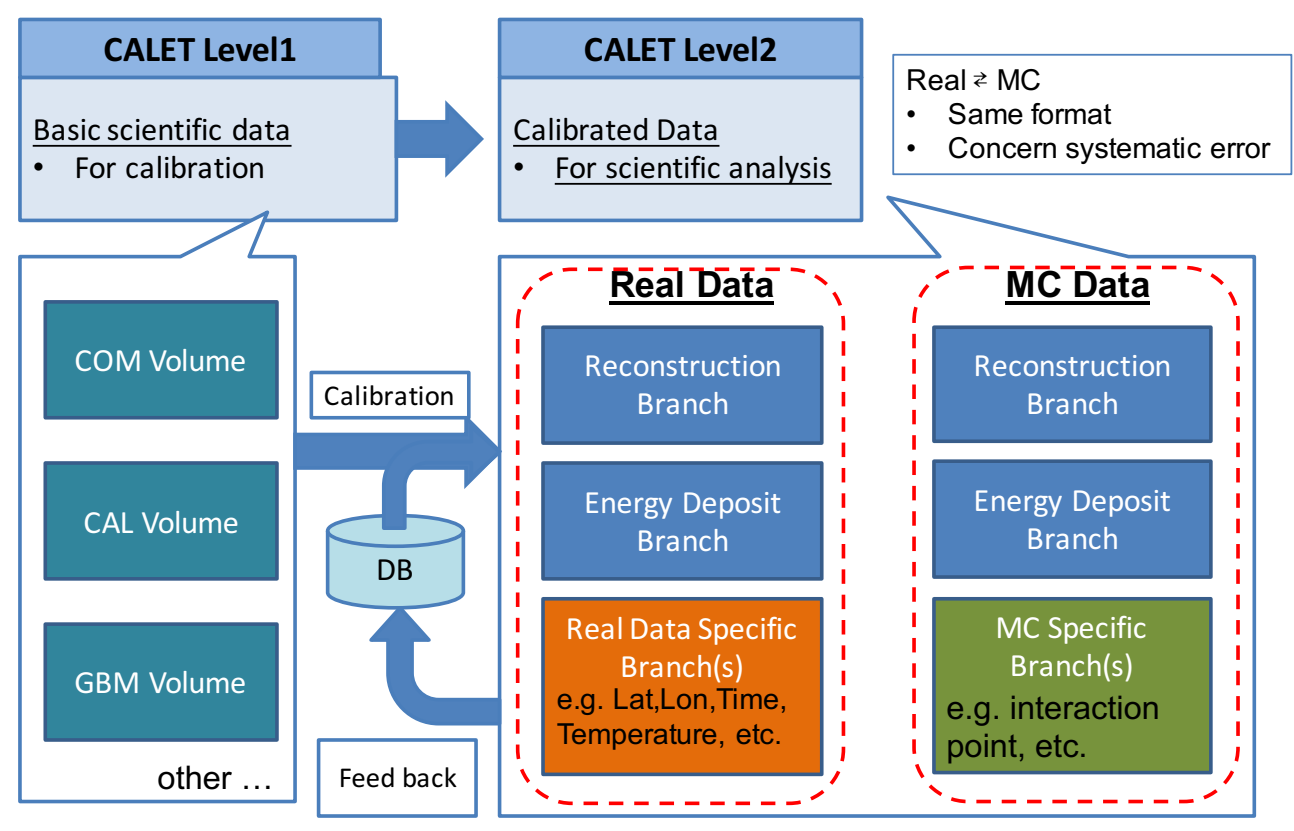

Figure 4: Data analysis flow for creating calibrated data (Level-2). The calibration parameters are stored in a data base and used for iterative improvements of calibration, event reconstruction, and MC data.

Various detector calibrations are performed using the CALET Level-1 data and Level-2 data for physics analysis[?]. Physics analysis is performed using CALET Level-2 in which flight data (FD) and Monte Carlo simulation data (MC) are equivalent in format and in data quality. In order to produce the Level-2 data set, the energy deposits of all of the channels, which are the basis of the Level-2 data, are first calculated by applying detector calibrations to FD and by considering detector responses in MC. In the process of Level-2 data production, the energy deposit array, which is equivalent between FD and MC, is fed into track reconstruction and energy reconstruction, and the various variables for event selection are calculated in both FD and MC. Since we use the flexible format, where it is possible to record the results of multiple algorithms in Level-2 data, 
comparisons between different algorithms can be easily carried out. By taking advantage of such Level-2 data features, efficient and detailed study of systematic uncertainty becomes feasible.

The calculation nodes at WCOC have over 700 cores working at present, processing the high level flight data and creating MC data. Spectral data and subsets of data for individual science targets will be called Level-3 data or higher. The data analysis using each data set will be carried out independently in each institute, and the official dataset to be used for publication is processed at WCOC.

\section{Summary}

CALET is a long-term high energy cosmic ray observation mission and aims to make unique observations by extending the previous limits of direct measurements. It is important to continue stable operation, and stable operations have continued since they began in October 2015. Figure
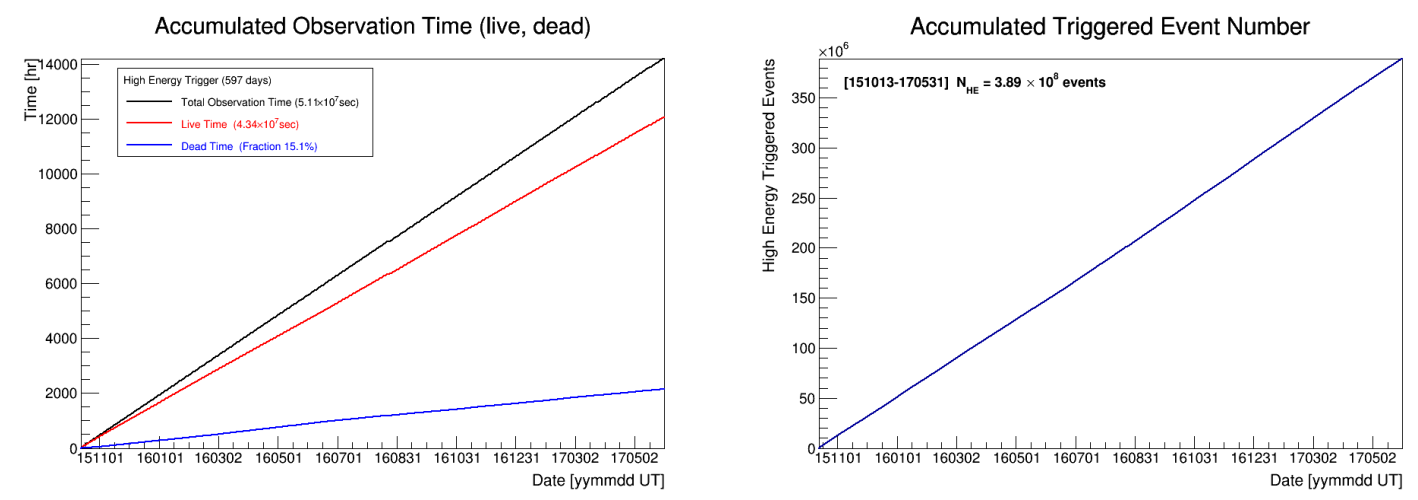

Figure 5: Left figure is an accumulation of observation time (black line). The red and blue line are live time and dead time. Total live time reaches $4.43 \times 10^{7} \mathrm{~s}$. Right figure is an accumulation of HE triggered events. Total number of HE events through the end of May 2017 is $3.89 \times 10^{8}$.

?? shows the accumulation of observational live time and HE triggered events. Since October 2015, observation time has increased smoothly without trouble. The transmission of data from JAXA-GSE to WCOC and processing the data for scientific analysis at WCOC has also proceeded smoothly. For ensuring continued stable operation, we are improving the monitoring system with the goal of alerting us immediately to any sudden change.

Meanwhile processing data for scientific analysis, distribution of science data, and science analysis of CALET observations are progressing smoothly.

\section{Acknowledgements}

We gratefully acknowledge JAXA's contributions to the development of CALET and to the operations on board the ISS. We also wish to express our sincere gratitude to ASI and NASA for their support of the CALET project. Finally, this work was partially supported by a JSPS Grant-in-Aid for Scientific Research (S) (no.26220708) and by the MEXT-Supported Program for the Strategic Research Foundation at Private Universities(2011-2015) (no.S1101021) at Waseda University. 


\section{References}

[1] S. Torii for the CALET Collaboration, "Calorimetric Electron Telescope (CALET) for the International Space Station", Proceedings of the 34th International Cosmic Ray Conference (The Hague, Netherland), 581 (2015)

[2] Y. Asaoka et al., "Development of the Waseda CALET Operations Center (WCOC) for Scientific Operations of CALET", Proceedings of Science, Proc. of the 34th ICRC (The Hague, Netherlands),603 (2015).

[3] Y. Asaoka et al., "Energy Calibration of CALET Onboard the International Space Station", Astropaticle Physics. 91 (2017) 1.

[4] B. Rauch et al., "Status of the CALET Ultra Heavy Cosmic Ray Analysis" , Proceedings of Science, Proc. of the 35th ICRC (Busan, Korea), 180 (2017) 